\section{Nature's Engineering Marvels: the Structure and Chemistry of a Butterfly Wing}

\author{
V.S. Smentkowski, S.G. Ostrowski, E.J. Olson, J.
}

Cournoyer, K. Dovidenko, R.A. Potyrailo General Electric Global Research Center, Niskayuna, NY smentkow@research.ge.com

Much effort is currently being expended in nanotechnology and other fields to build biomimetic, or nature-inspired, materials. The first step in this process is often to develop a more complete understanding of the structure and chemistry of biological systems. In this article, we will compare and contrast data collected on a common biological sample, a butterfly wing, using a variety of analytical techniques. Transmission Electron Microscopy (TEM) was used in order to perform bright field imaging of the sample cross section; Light Microscopy (LM) and Scanning Electron Microscopy (SEM) were used to provide structural information of the outer wing surface at various magnifications; Time-of-Flight Secondary Ion Mass Spectrometry (ToF-SIMS) was used in order to image the chemical composition of the outer

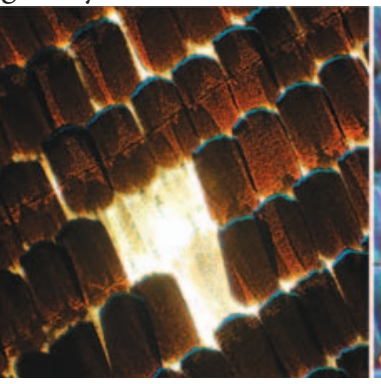

Figure 2. Transmitted light (left), reflected light (middle), and fluorescence light (right) micrographs of a Morpho butterfly wing. spectrum of the top butterfly wing surface is shown in Figure 3 . The fluorescence spectrum was collected using $355 \mathrm{~nm}$ excitation, and was not corrected by the spectral response of the optical system.

Light microscopy allows for rapid evaluation of butterfly wings with minimal sample preparation in air (and also under gaseous and/or liquid environments). Fluorescence spectra provides indirect chemical information (the analyst detects the energy of emitted photons, correlates the photon energy and peak shape with emission spectra of known fluorophores, and then extracts chemical information).

Secondary Electron (SE) images of the Morpho butterfly wing were collected using a Zeiss Supra 55VP SEM. A small section most surface layer; and Focused Ion Beam (FIB) techniques were used to cut (micro machine) features into the wing. Each of these analytical techniques has sample preparation and data collection challenges that will be summarized and compared. We will dem-

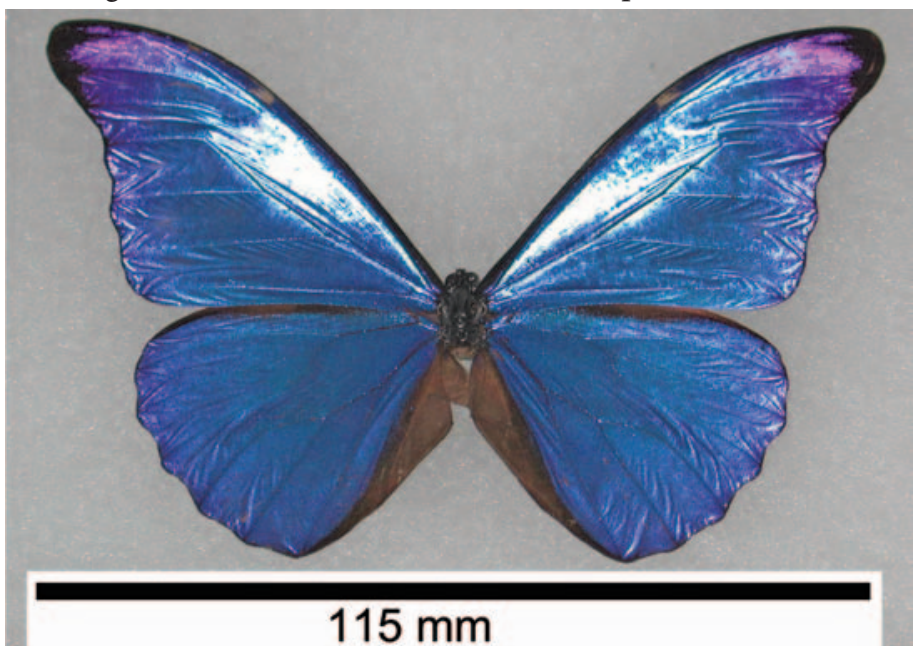

Figure 1. Optical photograph of a Morpho Menelaus butterfly wing.

onstrate that these analytical techniques provide complimentary information that helps the researcher understand the sample [1].

Figure 1 shows a photo of a Morpho Menelaus butterfly. Light microscopy measurements were performed using an Olympus BH-2 microscope coupled to an Ocean Optics Model ST2000 spectrofluorometer to measure the fluorescence spectra. Figure 2 shows transmitted light (left), reflected light (middle), and fluorescence light (right) micrographs revealing the layout of the scales, the reflectivity of the scales, and the native florescence of the scales. The three bright scales in the transmission image (left), and the three gold colored scales in the reflectance image (middle), correspond to regions of the wing that have two or more layers of scales. The reflectance image reveals the spectral and spatial distribution of light produced by the structural color of the scales [2]. A fluorescence of the wing was attached to an SEM pin stub using silver paint. A thin Pt coating (close to a monolayer) was sputtered onto the sample in order to minimize sample charging. Charging artifacts were further minimized by using a rapid scan rate. Figure 4 shows $\mathrm{SE}$ images taken at $3 \mathrm{kV}, 0^{\circ}$ tilt, and various magnifications. The low magnification SE image (Figure 4, top) provided an overview of the cover and ground scales, the higher magnification images (middle and bottom) provide additional detail regarding the ridge, lamellae, and microrib structures.

An FEI NOVA 200 Dual Beam FIB-SEM instrument was used to cut (micro machine) features into a wing scale. Figure 5 shows SE images collected in the FIB-SEM instrument on a butterfly wing that has finer microrib structures (than present in Morpho butterfly wings). The top panel shows low magnification SE images of the FIB cut at a sample tilt of $52^{\circ}$. The bottom panel shows high magnification SE images prior to and after FIB cutting (left, $0^{\circ}$ tilt; right $52^{\circ}$ tilt respectively).

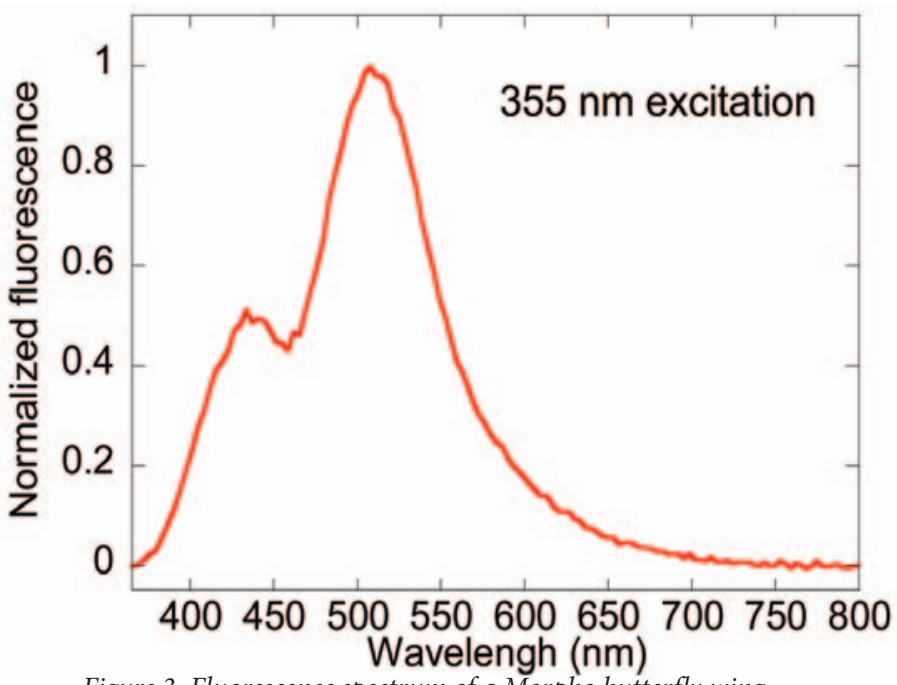

Figure 3. Fluorescence spectrum of a Morpho butterfly wing. 


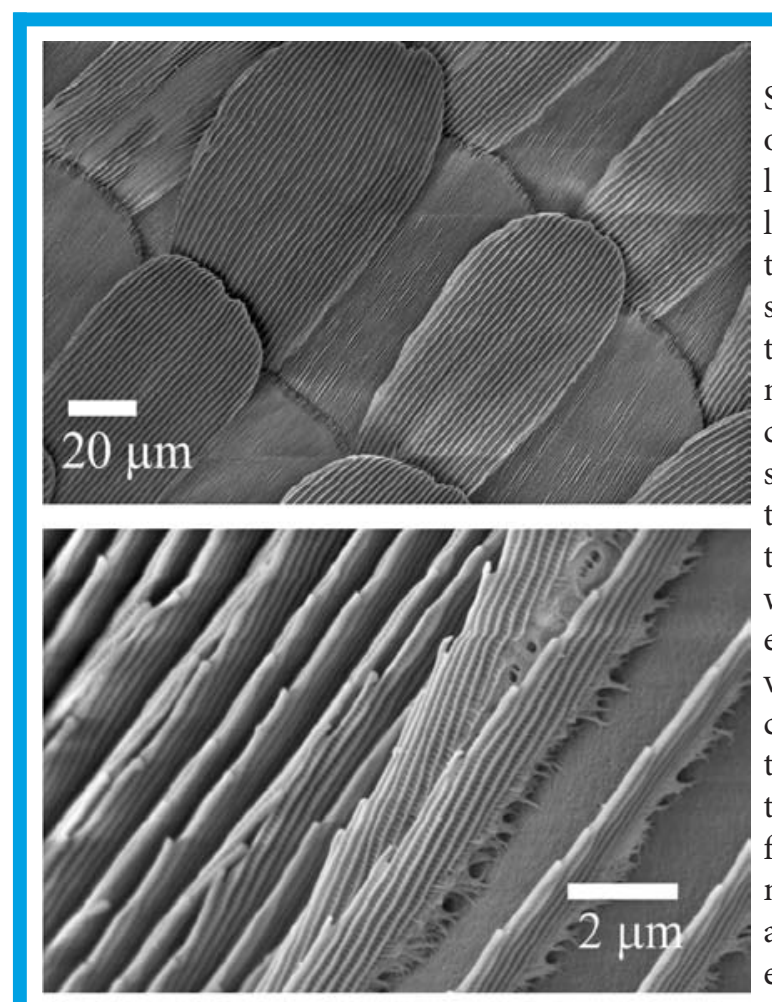

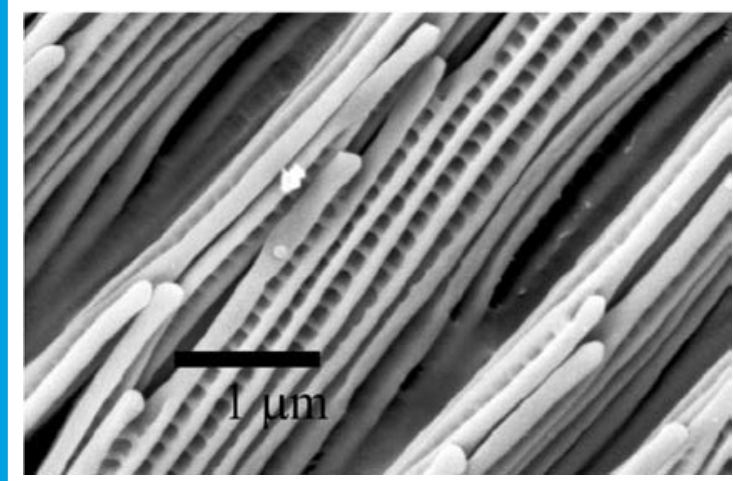

Figure 4. SE images of a Morpho butterfly wing taken at $3 \mathrm{kV}, 0^{\circ}$ tilt, and various magnifications.
FIB-SEM, the Pt thickness was slightly greater than for SEM. FIB milling was carried out using low beam currents (10 to $30 \mathrm{pA})$ in order to minimize damage of the delicate wing structures. Moderate sample preparation is needed for both SEM and FIB-SEM analysis.

TEM was used to perform bright field imaging of the Morpho butterfly wing cross section. The TEM analysis was performed using a FEI CM 100 instrument operating at $100 \mathrm{kV}$ accelerating voltage. Sections of butterfly wings were coated with Pt in order to enhance contrast. The Pt coated wing was then mounted in epoxy and microtomed at room temperature (thickness $\sim 80 \mathrm{~nm})$. TEM images are shown in Figure 6, the pillar structures are clearly visible.

Surface analysis of butterfly wings was performed using an ION TOF model ToF-SIMS IV instrument [3]. A small section of the wing was cut, laid flat onto the sample holder, and placed under a flat metal plate with $3 \mathrm{~mm}$ diameter holes. A low energy electron flood gun was used to stabilize the surface charge during analysis. ToF-SIMS images were collected at various magnifications (500 micron to 8 micron imaged areas). At each pixel in a ToF-SIMS image, an entire mass spectrum is saved. ToF-SIMS spectra provide both elemental informa-
SEM analysis of FIB cuts allows the analyst to view the internal structure through the micro-machined cross section. Sections of butterfly wings were mounted using silver paint. $\mathrm{Pt}$ coating protocols similar to those used for SEM (as mentioned above) were employed; for
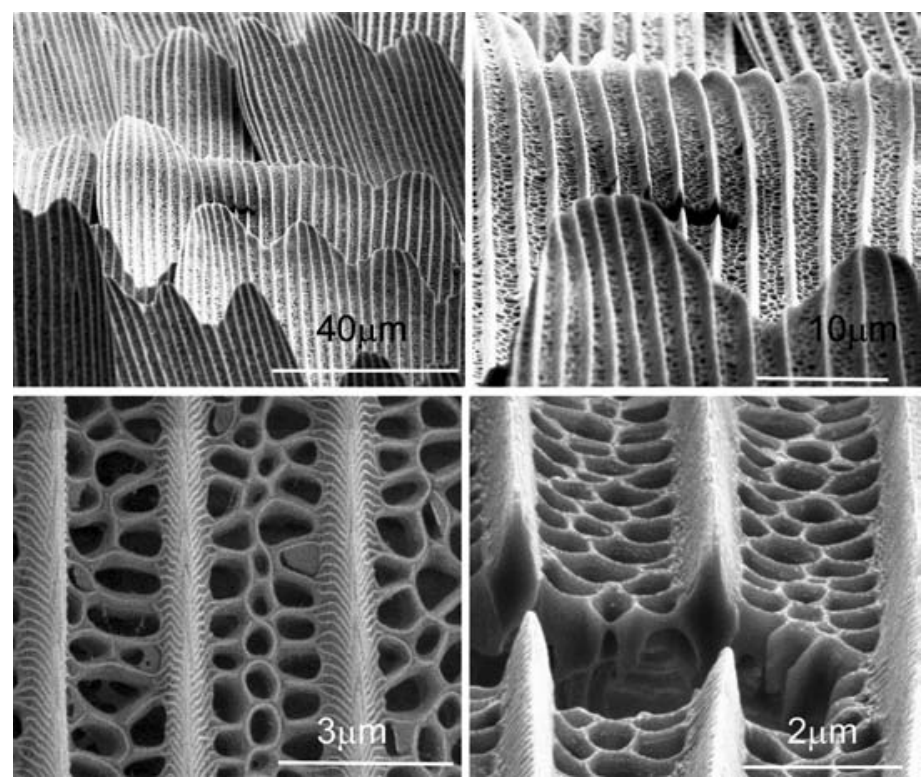

Figure 5. SE images collected in the FIB-SEM instrument. The top panel shows low magnification images of the FIB cut at a sample tilt of $52^{\circ}$. The bottom panel shows high magnification images prior to and after FIB cutting (left, $0^{\circ}$ tilt; right $52^{\circ}$ tilt respectively).

tion (all elements including $\mathrm{H}$ ) as well as high mass molecular peaks (lipids, proteins, etc). $\mathrm{A} \mathrm{Bi}_{3}{ }^{+}$cluster primary ion beam was used for the analysis shown here in order to increase the yield of high mass species [3]. ToF-SIMS spectra contain thousands of peaks and advanced multivariate statistical analysis (MVSA) tools are useful for converting the massive amount of information into a smaller number of chemical components (which are represented by a spectral signature and an image showing the lateral distribution of each chemical component) [4]. We are using the AXSIA based MCR algorithm developed at Sandia National Laboratories for MVSA analysis of ToF-SIMS data [4]. The left panel of Figure 7 shows colored overlays of component images generated at three magnifications from a section of the same butterfly wing analyzed by FIB-SEM (Figure 5). The right panel of Figure 7 shows the spectral signature of the 5 chemical components for the middle $(300 \mu \mathrm{m})$ image. These results demonstrate that the chemical composition of the native wing is not uniform.

Sections of Morpho butterfly wings were functionalized using metal porphyrin complexes. The right panel of Figure 8 shows the structures of the metal porphyrins used, as well as control ToF-SIMS spectra of the metal porphyrins (on a clean Si wafer). Note that, using ToF-SIMS, we are able to detect the intact metal porphyrin
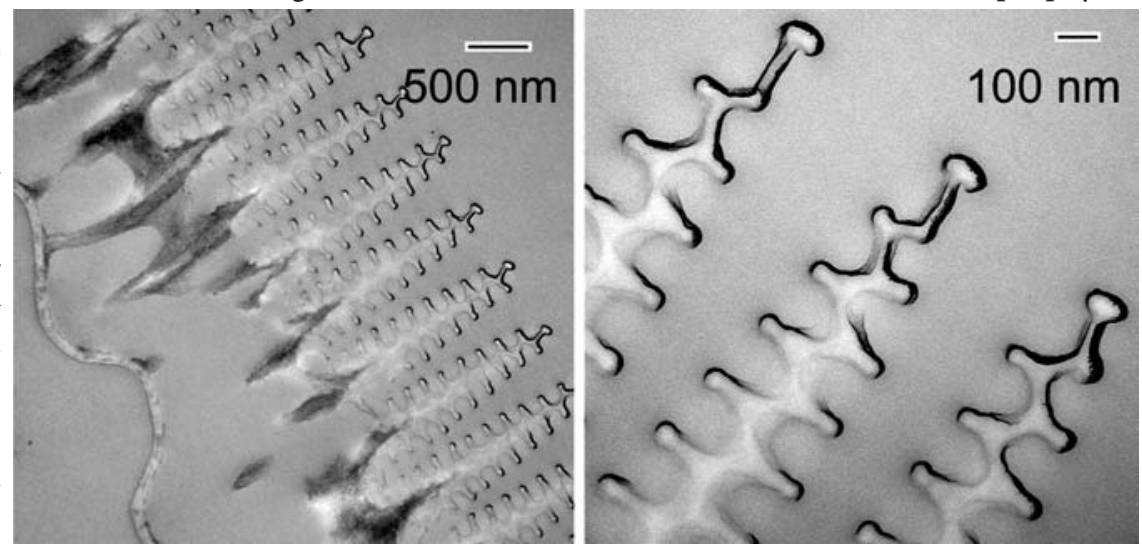

Figure 6. Bright field TEM images of Morpho butterfly wing cross section. 

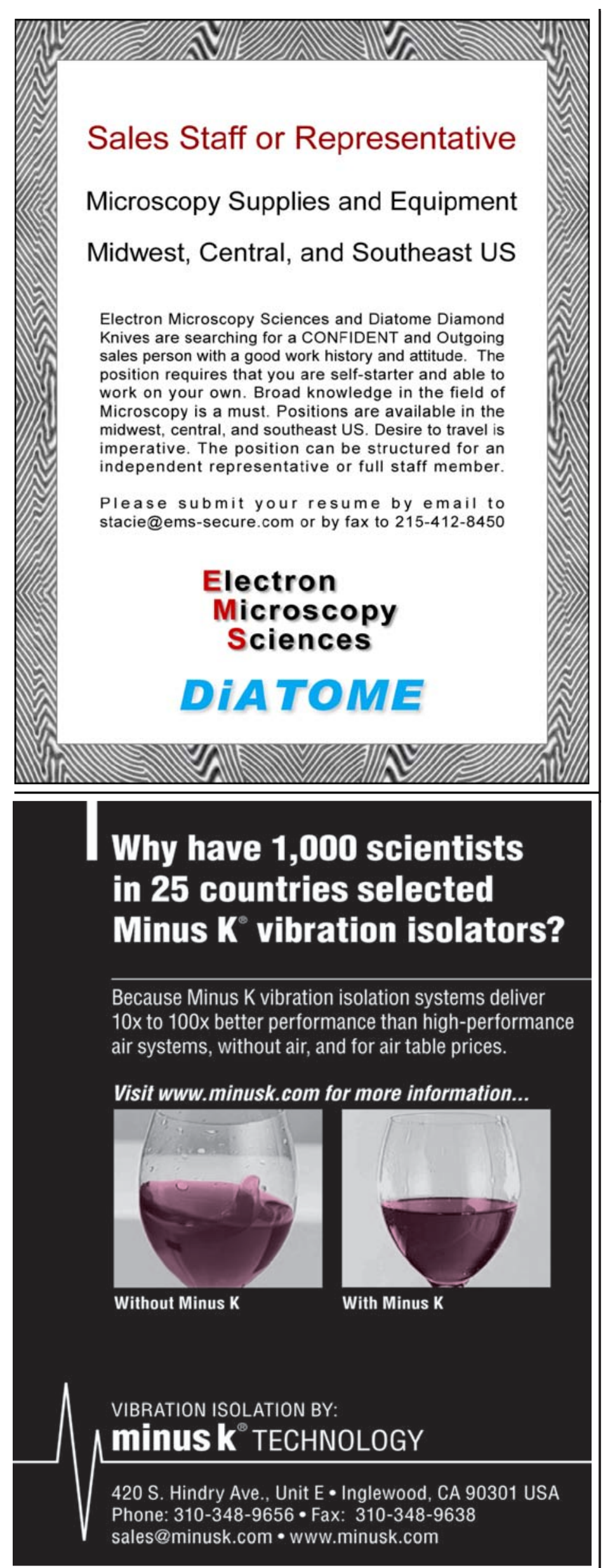

\section{The Leaders in Lift Out Technology}

\author{
Now selling $5^{\text {th }}$ Generation \\ Ex-Situ Systems
}

INTRODUCING THE WORLDS FIRST AUTOMATED EX-SITU LIFT OUT SYSTEM

\section{"THE AUTO LIFT"}

The new "Auto Lift" is unmatched in maximizing TEM Sample throughput. No other instrument or technique is faster when working with a FIB. Great for Clean Room Environments!

\section{The Ex-Situ Method by Micro Optics of Florida, Inc.}

- The "Original" Lift Out Technique

- The New "Auto Lift" for high throughput.(Easy Upgrade for Existing Customers)

- Allows your FIB to do what it does best: Make TEM Samples

- Proven, Fast, Easy Technology Over 100 sold worldwide

- Success Rate is 95\%

- Cost Effective Systems including: The New "Auto Lift", Standard One and Two Manipulator systems, and a University model

Be sure to specify our Lift Out with your FIB!

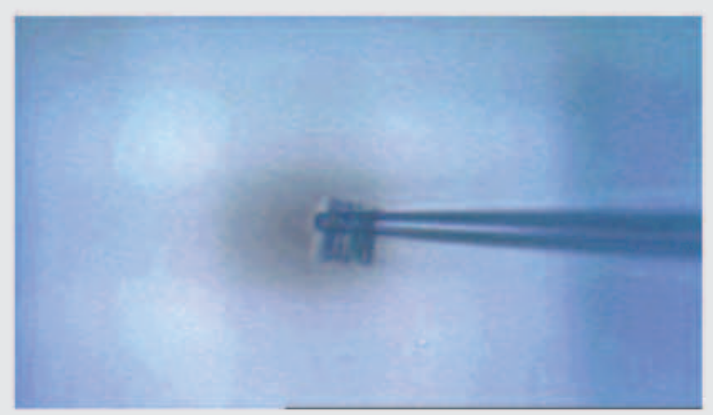

Micro Optics of Florida, Inc. (800) 545-3996 info@microopticsfl.com 

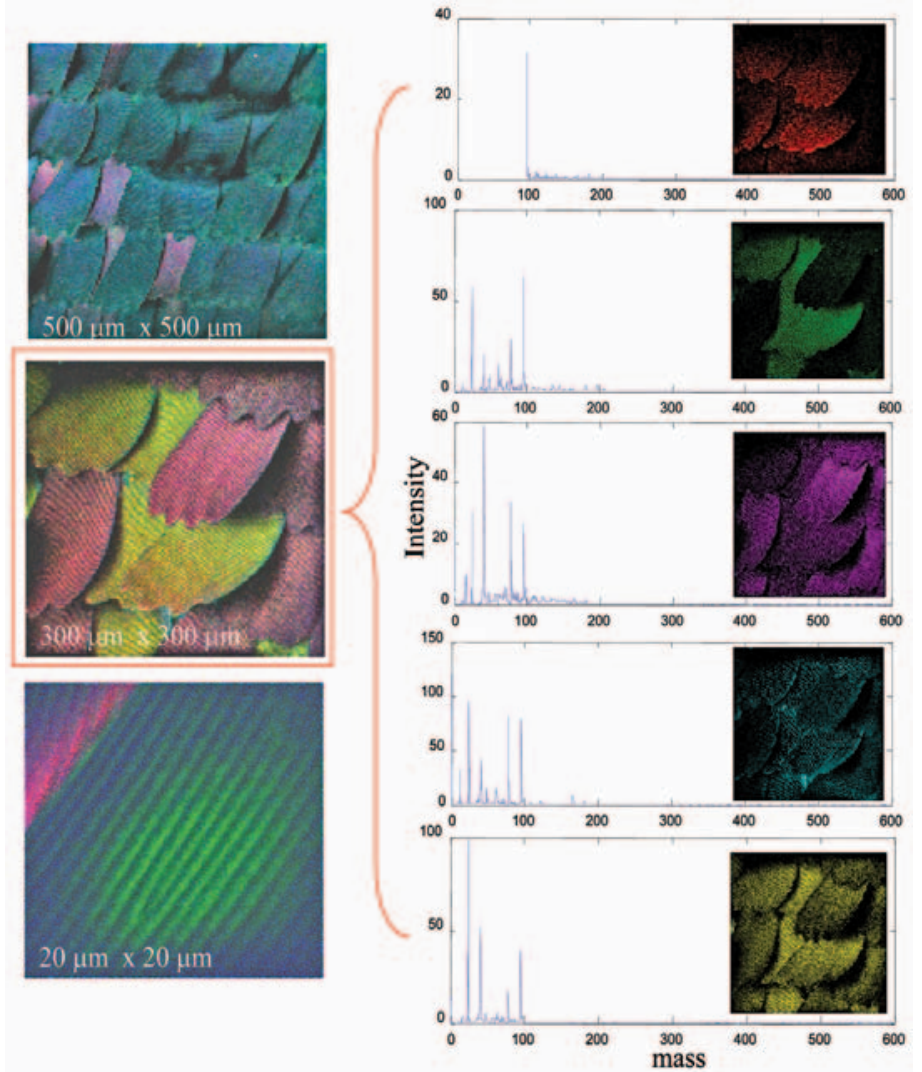

Figure 7. The left panel shows color overlays of MVSA component images generated from ToF-SIMS analysis (three magnifications). The right panel shows the spectral signature of the 5 MVSA components derived from the middle $(300 \mathrm{~mm}$ ) image.

complexes. For the Co-porphyrin (top panel, Figure 8) the Coporphyrin dimer species is detected at 1,718 amu. The ToF-SIMS images (left panel, Figure 8) show the lateral distribution of the metal porphyrins (both the intact parent species as well as lower mass fragment ions), species indicative of the unfunctionalized butterfly wing, and color overlays. It is noted that different porphyrins adhere to select regions of the wing, suggesting a chemical heterogeneity of the wing surface.

In this article, we show that different analytical techniques provide complimentary information that helps the researcher understand the sample.

1) Light Microscopy allows imaging at micron lateral resolution, and provides a cursory view of the wing with minimal sample preparation. The low native fluorescence of pigment molecules shows that the brilliant colors of the Morpho wing are based on structural features and not on pigments [2].

2) SE images reveal structural details of wing surface (ridge, lamellae, microribs).

3) FIB-SEM allows for micro-machining of delicate samples such as butterfly wings and also provides in-situ SEM imaging of the internal structures of the wing.

4) TEM provides bright field imaging of the interior structure (cross section) of the butterfly wing. The repeated, stacked pattern of microstructures cause the diffraction and interference of light and gives rise to the structural colors of the butterfly wing [2].

5) ToF-SIMS allows for the identification and imaging of the chemical composition and spatial distribution of species such
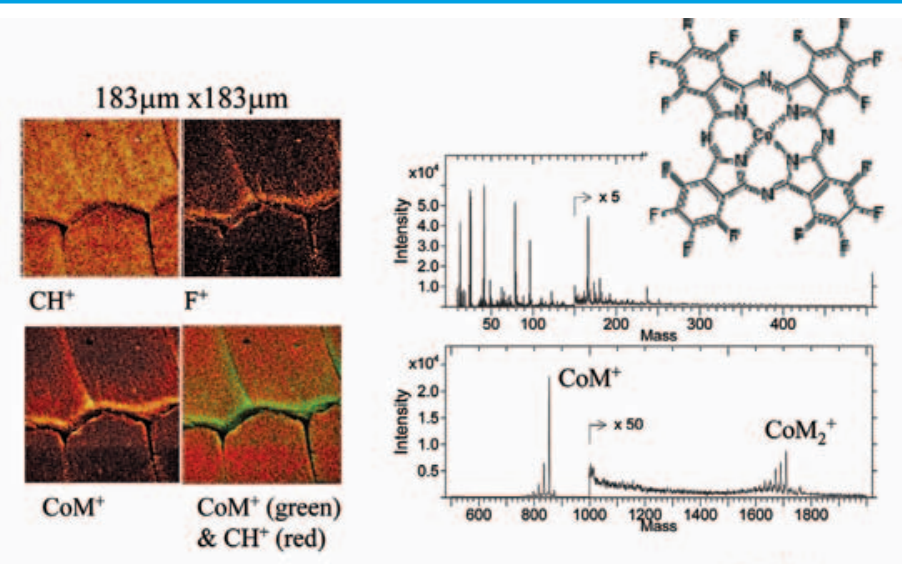

$150 \mu \mathrm{m} \times 150 \mu \mathrm{m}$
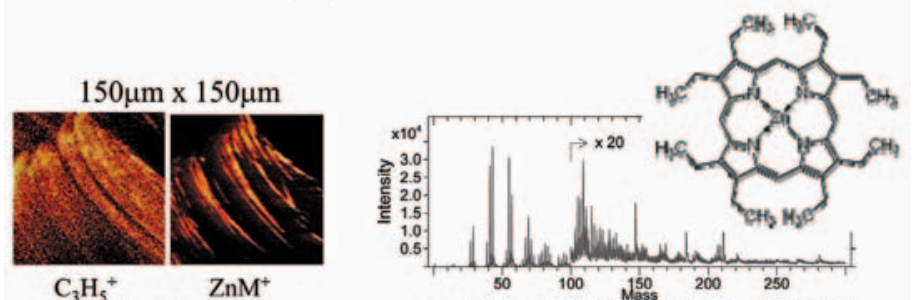

$\mathrm{C}_{3} \mathrm{H}_{5}^{+}$ $\mathrm{ZnM}^{+}$

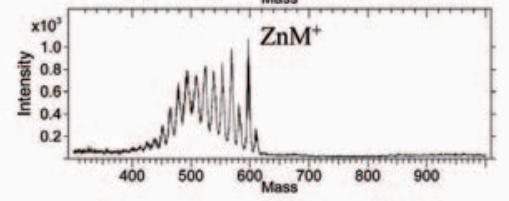

$\mathrm{ZnM}^{+}$(green) \&

$\mathrm{C}_{3} \mathrm{H}_{5}{ }^{+}$(red)

Figure 8. Structures of metal porphyrins and control ToF-SIMS spectra of the metal porphyrins (right panel). The ToF-SIMS images (left panel) show the lateral distribution of the metal porphyrins, species indicative of the chemistry of the unfunctionalized Morpho butterfly wing, and color overlays.

as lipids, protein fragments, and metal poryphrin dyes. ToFSIMS images of the spatial distribution of chemically specific dyes highlights the different chemical environments present on the wing structures. MVSA statistical analysis tools are useful for converting the massive amount of chemical information into a handful of components.

We would like to thank our management team at General Electric Global Research Center for funding the analysis reported here.

\section{References}

[1] LM, SEM, TEM, and FIB techniques have been used to analyze butterfly wings. The focus of our work is to compare and contrast a larger sampling of microscopy techniques on the same sample.

For LM see: M. Srinivasarao et al., Chem. Rev. 99 (1999) 1935; S. Kinoshita et al., Chem. Phys. Chem. 6 (2005) 1442; P. Vukusic et al., Proc. R. Soc. Lond. B 266 (1999) 1403, P. Vukusic, Science 310 (2005) 1151.

For SEM see: S. Kinoshita et al., Proc R Soc Lond B 269 (2002) 1417.

For FIB see D.G. Stavenga et al., Proc. R. Soc. Lond. B 271 (2004) 1577.

For TEM see A. Argyros et al. Micron, 33 (2002) 483.

[2] S. Kinoshita, S. Yoshioka, "Structural Colors in Nature: The Role of Regularity and Irregularity in the Structure", Chem Phys Chem., 6 (2005) 1442.

[3] ION-TOF GmbH, Gievenbecker Weg 15, 48149 Muenster, Germany, http://www.ion-tof.com.

[4] M.R. Keenan, P.G. Kotula, J.R. Michael, Microsc. Microanal. 9 (2003) 1. 


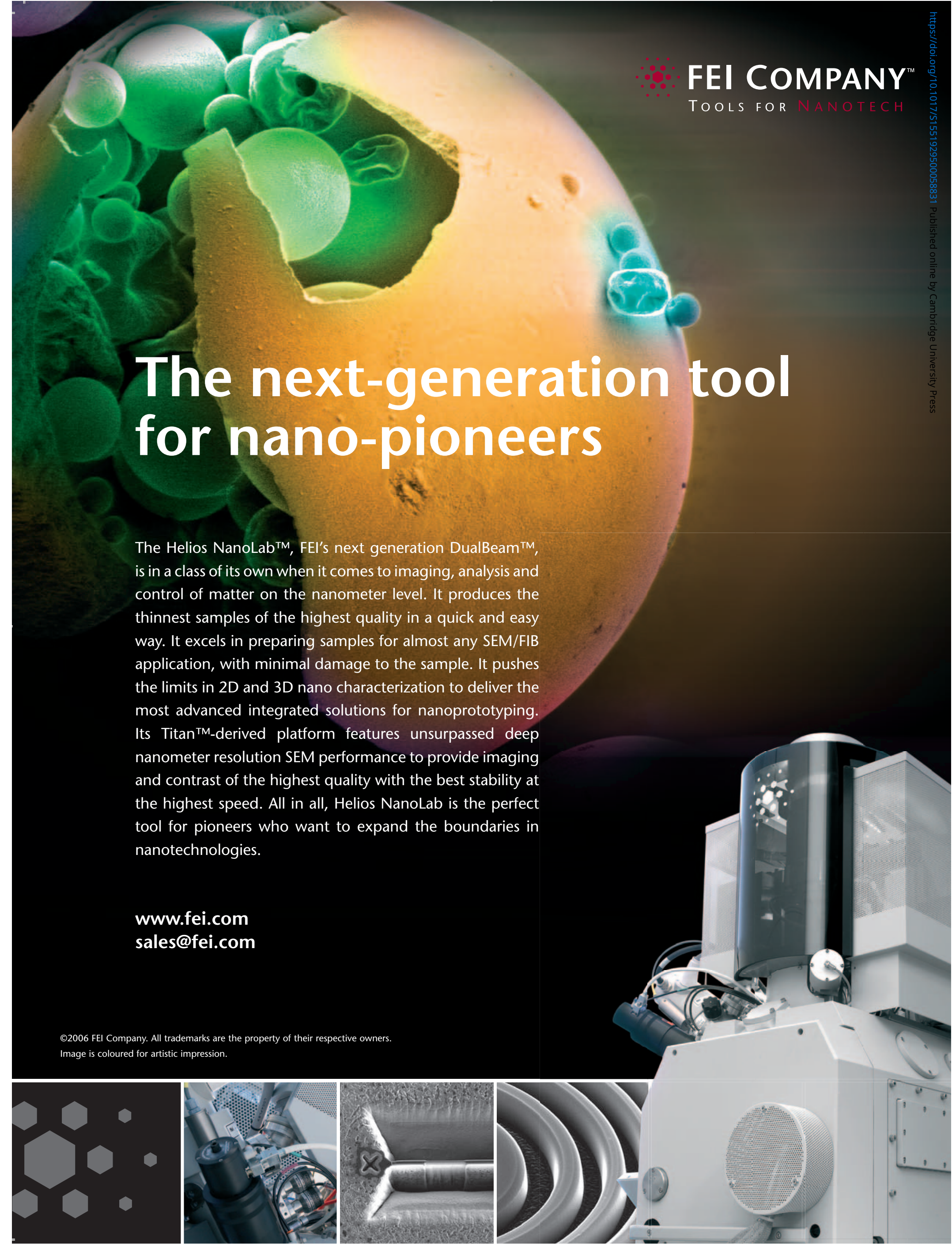

